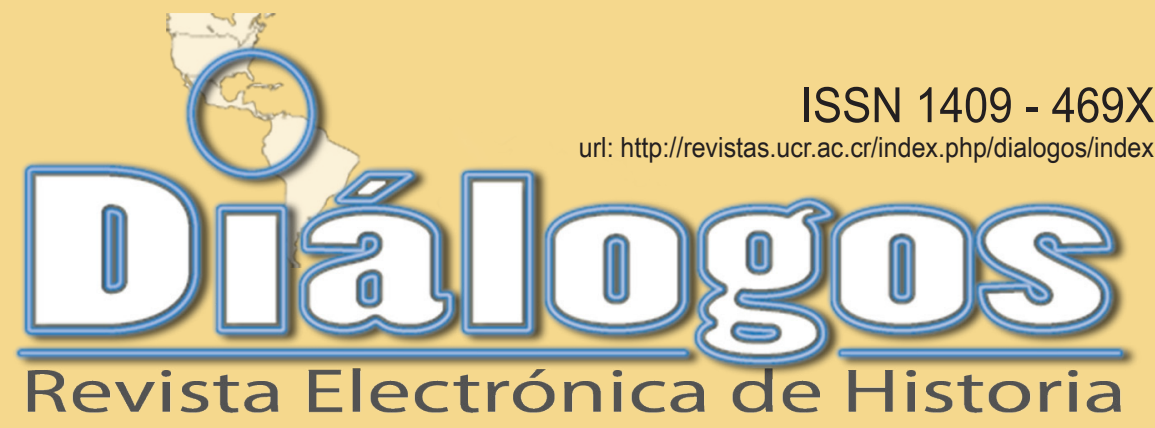

Escuela de Historia. Universidad de Costa Rica Volumen 15 Especial Región Occidente de Costa Rica - Octubre, 2014

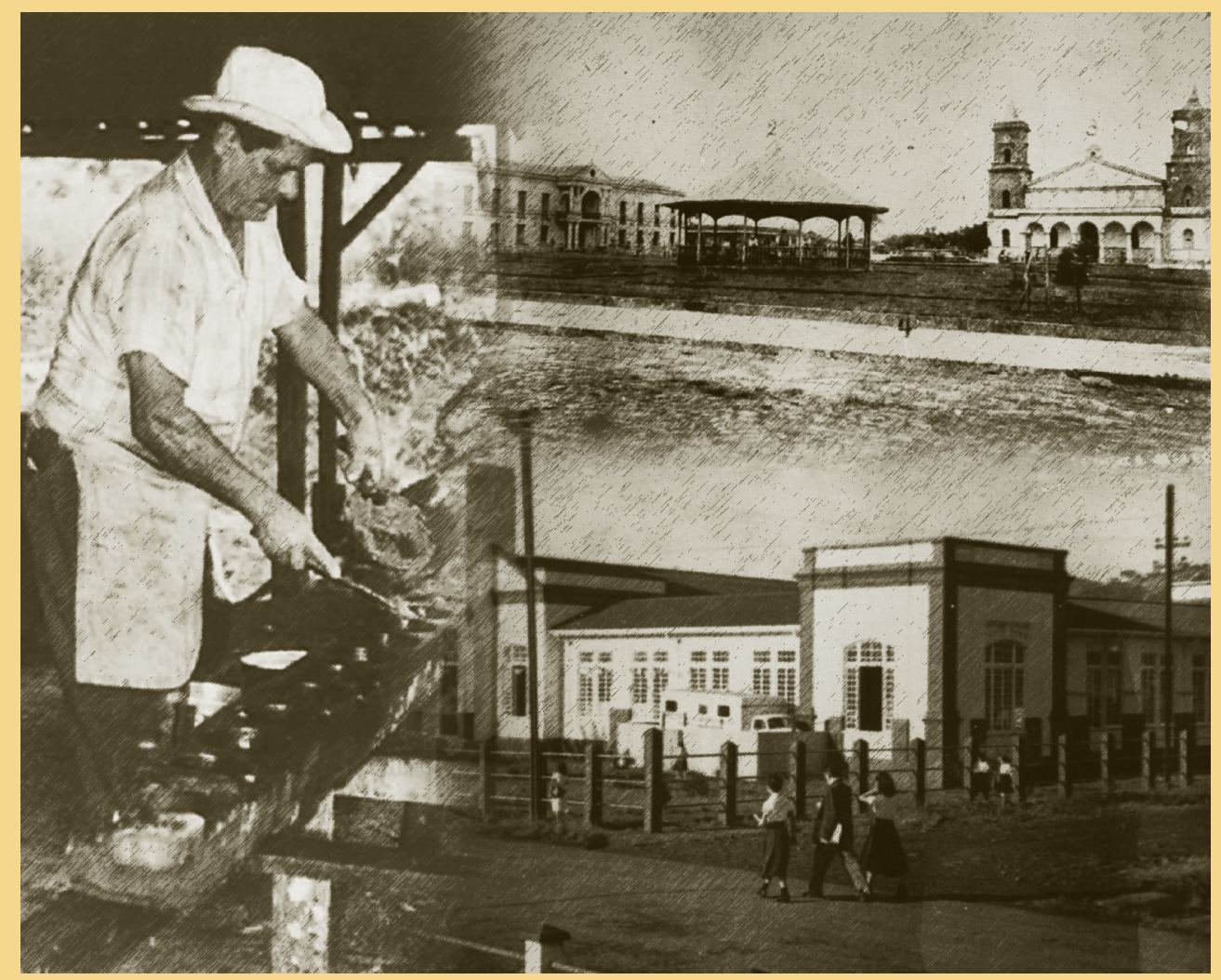

Director de la Revista: Dr. Juan José Marín Hernández juan.marinhernandez@ucr.ac.cr

Editor académico: Dr. David Díaz Arias - david.diaz@ucr.ac.cr

Editores invitados: M.Sc. William Solórzano Vargas - william.solorzano@ucr.ac.cr M.Ed. Maynor Badilla Vargas - mainor.badilla@ucr.ac.cr

Editora técnica: M.Sc. Marcela Quirós G. - marcela.quiros@ucr.ac.cr 
Dr. Juan José Marín Hernández, Catedrático. Director del Centro de Investigaciones Históricas de América Central. Universidad de Costa Rica. Costa Rica. juan. marin@ucr.ac.cr

Dr. David Díaz Arias: Catedrático. Historia Política, Director del posgrado de Historia y Docente de la Escuela de Historia, Universidad de Costa Rica, Costa Rica.david.diaz@ucr.ac.cr

Dr. Ronny Viales Hurtado. Catedrático. Historia Económica y Social. Universidad de Costa Rica. Director de la Escuela de Historia. Costa Rica. ronny. viales@ucr.ac.cr

MSc. Francisco Enríquez. Historia Social. Universidad de Costa Rica. Costa Rica. francisco.enriquez@ucr. ac.cr

Dra. Ana María Botey. Historia de los movimientos sociales. Universidad de Costa Rica. Costa Rica. abotey@gmail.com

\section{Miembros del Consejo Asesor Internacional:}

Dr. José Cal Montoya. Universidad de San Carlos de Guatemala. Guatemala. jecalm@correo.url.edu.gt

Dr. Juan Manuel Palacio. Universidad Nacional de San Martín. Argentina.jpalacio@unsam.edu.ar

Dr. Eduardo Rey. Universidad de Santiago de Compostela. España. ereyt@usc.es

Dr. Heriberto Cairo Carou. Departamento de Ciencia Política y de la Administración III - Universidad Complutense de Madrid. España. hcairoca@cps.ucm.es

Dra. Rosa de la Fuente. Departamento de Ciencia Política y de la Administración III Universidad Complutense de Madrid. España. rdelafuente@cps. ucm.es

Dr. Javier Franzé. Departamento de Ciencia Política y de la Administración III Universidad Complutense de Madrid. España. javier.franze@cps.ucm.es

Dr. Jaime Preciado Coronado Departamento de Estudios Ibéricos y Latinoamericanos. Universidad de Guadalajara. México.japreco@hotmail.com

Dr. Gerónimo de Sierra. Vicerrector de la Universidade Federal da Integração Latino-Americana (UNILA) y Departamento de Sociología, Facultad de Ciencias

Sociales de la Universidad de la República. Uruguay. geronimo@fcs.edu.uy

Dr. Antonio Palazuelos. Departamento de Ciencia Política y de la Administración III - Universidad Complutense de Madrid. España. palazuelosa@cps. ucm.es

Dr. Werner Mackenbach. Universidad Potsdam. Alemania.werner.mackenbach@uni-potsdam.de

Dr. Guillermo Castro. Ciudad del Saber Panamá. Panamá.gcastro@cdspanama.org

Dra. Natalia Milanesio. University of Houston. Estados Unidos.nmilane2@Central.UH.EDU

Dr. Ricardo González Leandri. Consejo Superior de Investigaciones Científicas - España. España. rgleandri@gmail.com

Dra. Mayra Espina. Centro de Estudios Psicológicos y Sociológicos, La Habana. Cuba.mjdcips@ceniai.inf.cu

Dra. Montserrat Llonch. Departamento de Economía e Historia Económica Universidad Autónoma de Barcelona. España. montserrat.llonch@uab.es

Dra. Estela Grassi. Universidad de Buenos Aires. Argentina. estelagrassi@gmail.com

Dra. Yolanda Blasco. Universidad de Barcelona. España. yolandablasco@ub.edu

Dr. Alfredo Falero. Departamento de Sociología. Universidad de la República. Uruguay. alfredof@adinet. com.uy

Portada:

Fotografía: ( Collage "Campesinos en la producción de dulce en un trapiche de Rincón de Mora, Ran Ramón, 1987" ; "Panorámica del Antiguo Palacio Municipal, Antigua Iglesia, Parque y Kiosco de San Ramón, hacia 1920; y Escuela Jorge Washinton, San Ramón hacia 1955). Estas fotografías pertenecen a la Colección Fotográfica del Museo Regional de San Ramón- UCR. Montaje fotográfico: Lic. Juan Gabriel Madrigal Cubero ).

\section{Equipo Técnico Editorial:}

Diagramación y

Edición técnica:

M.Sc. Marcela Quirós Garita. marcela.quiros@ucr.ac.cr

Soporte técnico: Kevin Trejos Vargas

Revisión filológica: Baruc Chavarría Castro 
"Diálogos Revista Electrónica de Historia" se publica desde octubre de 1999.

\section{Diálogos está en los siguientes repositorios:}

Dialnet

http://dialnet.unirioja.es/servlet/

revista?tipo_busqueda=CODIGO\&clave_revista $=3325$

\section{Latindex}

http://www.latindex.unam.mx/larga.php?opcion=1\&folio=12995;

\section{UCRindex}

http://www.revistas.ucr.ac.cr

\section{Scielo}

http://www.scielo.cll

\section{eRevistas}

http://www.erevistas.csic.es/

\section{REDALYC}

http://redalyc.uaemex.mx/src/inicio/FrmBusRevs2.jsp?iEdoRev=2\&cvepai=11;

\section{LANIC}

http://lanic.utexas.edu/la/ca/cr/indexesp.html;

Repositorio de Revistas Universidad de Costa Rica

http://www.latindex.ucr.ac.cr/

Directorio y recolector de recursos digitales del

Ministerio de Cultura de España

http://roai.mcu.es/es/inicio/inicio.cmo

DOAJ Directory of open access \& Hybrid journals

http://www.doaj.org/doaj?func=byTitle\&hybrid=1\&query=D

Biblioteca de Georgetown

http://library.georgetown.edu/newjour/d/msg02735.htm

Asociación para el Fomento de los Estudios Históricos en Centroamérica

http://afehc.apinc.org/index.php?action=fi_aff\&id=1774

Universidad de Saskatchewan, Canadá

https://library.usask.ca/ejournals/view/1000000000397982

Monografias

http://www.monografias.com/Links/Historia/more12.shtml

\section{Hispanianova}

http://hispanianova.rediris.es/general/enlaces/hn0708.htm

Universidad del Norte, Colombia

http://www.uninorte.edu.co/publicaciones/memorias/enlaces.htm

Universidad Autónoma de Barcelona

http://seneca.uab.es/historia/hn0708.htm

Repositorio Invenia - Gestión del Conocimiento http://www.invenia.es/oai:dialnet.unirioja.es:ART0000086144

\section{Enlace Académico}

http://www.enlaceacademico.org/biblioteca/

revistas-en-formato-digital-centroamerica/

\section{Electronic Resources}

http://sunzi1.lib.hku.hk/ER/detail/hkul/3987318

Revistas académicas en texto completo http://web.prw.net/ vtorres/

Diálogos se anuncia en las siguientes instituciones y sitios académicos:

Maestroteca

http://www.maestroteca.com/detail/553/dialogos-revista-electronica-de-historia.html

Biblioteca de Georgetown

http://library.georgetown.edu/newjour/d/msg02735.htm

Asociación para el Fomento de los Estudios Históricos en Centroamérica

http://afehc.apinc.org/index.php?action=fi_aff\&id=1774

Universidad de Saskatchewan, Canadá

https://library.usask.ca/ejournals/view/1000000000397982

Monografias

http://www.monografias.com/Links/Historia/more12.shtm

Hispanianova

http://hispanianova.rediris.es/general/enlaces/hn0708.htm

Universidad del Norte, Colombia

http://www.uninorte.edu.co/publicaciones/memorias/enlaces.html

Universidad Autónoma de Barcelona

http://seneca.uab.es/historia/hn0708.htm

Repositorio Invenia - Gestión del Conocimiento

http://www.invenia.es/oai:dialnet.unirioja.es:ART0000086144

Enlace Académico

http://www.enlaceacademico.org/biblioteca/

revistas-en-formato-digital-centroamerical

\section{Electronic Resources}

http://sunzi1.lib.hku.hk/ER/detail/hkul/3987318

Revistas académicas en texto completo http://web.prw.net/ vtorres/

Diálogos Revista de Historia está catalogada por Sherpa Romeo como una revista verde.

La revista electrónica Diálogos es financiada por Vicerrectoría de Investigación de la Universidad de Costa Rica

Citado en: Scielo Dialnet - eRevistas - UCRindex Latindex - REDALYC - DOAJDirectorio y recolector de recursos digitales del Ministerio de Cultura de España

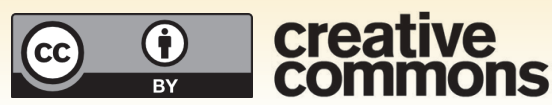




\title{
EDUCACIÓN CONTINUA EN PROMOCIÓN DE LA SALUD, DESDE UNA ACCIÓN INTERINSTITUCIONAL
}

\author{
CONTINUING EDUCATION IN PROMOTING HEALTH FROM AN \\ INTERINSTITUTIONAL ACTION
}

\author{
Ana Cristina Quesada Monge \\ Lidia Isabel Picado Herrera
}

\begin{abstract}
Palabras clave
Historia local, metodología histórica, historia local académica, historia local no académica.
\end{abstract}

\section{Keywords}

Local history, historical methods, academic local history, nonacademic local history.

Fecha de recepción: 7 de febrero, 2014 - Fecha de aceptación: 1 de junio, 2014

\begin{abstract}
Resumen
Si bien existe consenso en cuanto a la definición de promoción de la salud, no sucede lo mismo sobre su enfoque, pues varios autores han identificado diferencias en cuanto a la forma de visualizar su implementación o abordaje. En consecuencia, pese a que se ha reconocido su trascendencia, han existido dificultades para que su forma de operar no se centre en los paradigmas de corte biologistamédico-asistencialista en las instituciones y organizaciones que proveen servicios en salud, dado que esto genera que las acciones sigan orientándose a la prevención de la enfermedad. Mediante este artículo se pretende exponer los referentes teóricos generales y regionales, que motivaron la generación de dos proyectos de extensión docente ejecutados, así como las metodologías y los alcances obtenidos en el desarrollo de estos.
\end{abstract}

\begin{abstract}
Abstrac
There is a consensus about the definition of promoting health but not about its approach since many authors have identified differences with regard to its implementation and how to address it. As a consequence, there have appeared difficulties for it to operate not only focusing on the biologistmedical-welfare paradigms because this makes actions to be guided only to the prevention of disease. In this paper, we present the general and regional theoretical references that produced two projects of teaching extension and the methodologies and results of these projects.
\end{abstract}




\section{ASPECTOS TEÓRICOS QUE FUNDAMENTAN LA CREACIÓN DE UNA NUEVA LÓGICA DE ACTUACIÓN CON EL ENFOQUE EN PROMOCIÓN DE LA SALUD}

Para poder comprender el enfoque citado, es necesario hacer primeramente una referencia muy general respecto a ciertas conceptualizaciones sobre salud y en especial al proceso salud-enfermedad donde cobra relevancia.

\section{Evolución histórica del abordaje de la salud-enfermedad}

El concepto de salud-enfermedad ha sido objeto de estudio e interpretaciones desde diversos enfoques teóricos, pasando a lo largo de la historia de conceptos o visiones mágico, religioso, biologista y psicológicas a posiciones más de índole social. Para efectos de este artículo, se centrará el estudio en dos posiciones teóricas sobre la salud-enfermedad, a saber: la biologista y la sociológica.

Según Carmona, Rozo y Mogollón (2005), el enfoque biologista concentra su interés de intervención en el cuerpo. Es decir, el cuerpo es considerado como un aparato mecánico y un ente receptor de acciones y sustancias que pueden modificar su estado de salud y enfermedad. En tal sentido, el ser humano es reducido a la suma de órganos, dado que a este enfoque le interesa trabajar solo con las personas que manifiesten problemas físicos. Por otra parte, este enfoque considera la salud como estado de equilibrio, mientras que la enfermedad es vista como desequilibrio y anormalidad. De igual forma, abandona todo tipo de visiones humanistas en los procesos de intervención y actuación con el ser humano.

A raíz de esta debilidad que presenta el enfoque en mención, surge en los últimos años, la propuesta de valorar la salud desde las posturas Sociológicas, lo cual está cobrando fuerza e importancia en el contexto actual. En este caso, se determina que "la salud no es sencillamente la ausencia de enfermedad, es algo positivo, una actitud alegre hacia la vida y la aceptación entusiasta de la responsabilidad que la vida impone a la persona" (Carmona, Rozo y Mogollón, 2005, p. 67).

A la luz de este enfoque, diversas entidades internacionales empiezan a construir nuevos conceptos de salud. Una de estas instancias es la Organización Mundial de la Salud, que la define como: "completo estado de bienestar físico, mental y social y no solamente como ausencia de enfermedad" (Carmona, Rozo y Mogollón, 2005, p. 67). No obstante, según las investigadoras citadas anteriormente, este concepto presenta dos importantes limitaciones: la concepción de ver la salud como un estado tautológico, ideal, utópico, estático y ahistórico, donde su acepción sigue centrada en los individuos y no en problemas que se manifiestan en la colectividad. A pesar de que se trabaja el componente social, este sigue confundiéndose con los factores ambientales, que influyen en las condiciones de vida del ser humano. 
Ante la carencia de conceptos y respuestas más efectivas para el abordaje del proceso salud-enfermedad, surge en los últimos años la necesidad de visibilizar e integrar los vínculos sociales y los procesos de producción social en dicho abordaje. Es decir, la salud y la enfermedad son manifestaciones de los vínculos y relaciones sociales que construye el ser humano con su entorno; por consiguiente, la salud se valora de la siguiente manera (Carmona, Rozo y Mogollón, 2005, p. 68):

- No como una expresión antagónica, sino como la manifestación de los determinantes contextuales y personales que influyen en el sujeto a lo largo de la vida.

- $\quad$ Como un proceso continuo de actualización de las necesidades y posibilidades de desarrollo de los sujetos, en un contexto específico, lo que denota un proceso de permanente movimiento y cambio.

- $\quad$ Se teje en lugares y contextos específicos. Tiene un carácter histórico.

- $\quad$ La enfermedad debe ser abordada no como una situación individual del ser humano, sino como un proceso colectivo. Es decir, lo biológico está condicionado por lo social.

- La persona es vista de manera integral, es la integración de factores biológicos, psicológicos y sociales.

- La salud no puede ser vista como un fin en sí misma, sino como un recurso de la vida cotidiana, que capacita a las personas para alcanzar las aspiraciones individuales, sus necesidades estratégicas y cambiar el ambiente. Por tal motivo, no se puede apartar el componente cultural de la salud, donde se da la actualización permanente de dos estadios, el bienestar y la enfermedad, y su coexistencia está determinada por las condiciones de vida de las personas, pero también de las sociedades en su totalidad.

Esta perspectiva teórica del proceso salud-enfermedad que proponen Carmona, Rozo y Mogollón (2005), tiene grandes coincidencias con la propuesta que establece Breilh (1994). Este autor concibe la salud como un proceso y no como un estado asignado, dado que las responsabilidades en la construcción de la salud y del mejoramiento de las condiciones de vida, no solo está en las personas de manera individual, sino en la sociedad en su forma de organización colectiva.

Para Breilh (1994), la salud incluye todas las áreas de la vida de las personas y su bienestar está en función de la adecuada satisfacción de las necesidades individuales y sociales e induce abordarla de manera sectorial e intersectorial y mediante el compromiso social integrado al desarrollo de la sociedad; ello por cuanto la misma depende del esfuerzo coordinado de los sectores, de equipos interdisciplinarios, intersectoriales e interorganizacionales, que avancen hacia la efectiva construcción y aplicación de un modelo de salud. Por este motivo, la salud se ve como el resultado de una construcción colectiva. 
En concordancia con lo antes planteado, el Ministerio de Salud de Costa Rica, ha construido un modelo de la producción social de la salud, el cual incorpora cuatro factores básicos de acción, a saber:

- Factores biológicos: se refiere a todos aquellos elementos de salud, tanto física como mental, que se desarrollan dentro del cuerpo humano como consecuencia de la biología básica y de aspectos orgánicos del individuo. Por ejemplo: la carga genética, el proceso de envejecimiento, los sistemas internos del organismo, entre otros.

- Factores ambientales: incluye los relacionados con el hábitat humano. Se considera en este grupo las condiciones de saneamiento básico y de vivienda, el medio laboral, el escolar y otros.

- Factores socioeconómicos y culturales: incluye los estilos de vida, las decisiones, los hábitos, el ingreso económico, el nivel educativo, los aspectos culturales, el empleo, la recreación y la participación política.

- Factores relacionados con servicios de salud de atención a las personas: aspectos vinculados con el acceso, la cantidad, la calidad, la oportunidad y la organización de estos servicios de salud. (Dirección General de Salud, Ministerio de Salud, 2006, pp. 7-8)

En el marco del proceso salud-enfermedad en mención, se determinan tres escenarios de actuación: salud, riesgo e intervención o atención. De los tres escenarios, el primero es donde se posiciona la actuación del enfoque de promoción de la salud, mientras que en el segundo se ubica la prevención de la enfermedad.

Cuando se estudia la promoción de la salud, es posible identificar también varias acepciones en la forma de cómo hacerla efectiva. Para efectos prácticos se hará mención general de las principales perspectivas con las que se ha trabajado al respecto, para luego enfatizar en el enfoque que las autoras de este artículo han venido impulsando como parte de su quehacer institucional, en procura no solo de clarificar sus diferencias con el de prevención de la enfermedad, sino también de generar un cambio en el paradigma sobre la forma de cómo actuar desde, por y para la salud, a partir del enfoque de promoción de la salud con el fin de construir y dar prioridad a la cultura salutogénica.

\section{Los enfoques teóricos que se han construido alrededor del tema de la promoción de la salud}

Al igual que del concepto de salud, alrededor de su promoción se han construido diversos enfoques, que han sido identificados por varios autores; no obstante, para efectos de este trabajo, se resaltarán los enunciados por Carmona, Rozo y Mogollón (2005), así como de Eslava (2006). 
Según Carmona, Roza y Mogollón (2005), en la actualidad es posible identificar dos enfoques teóricos y metodológicos referentes a promover la salud. El primer enfoque es denominado "Estilos de vida saludables", que se vincula con las condiciones o formas de vida, actitudes o comportamientos del ser humano, de igual forma con el autocuidado, por lo que la responsabilidad de promover estos estilos de vida queda en manos de las instituciones prestadoras de los servicios en salud.

Otro enfoque que surge sobre el tema en cuestión, es denominado como " $\mathbf{L a}$ capacidad de potenciar el Desarrollo Humano". Este enfoque pretende favorecer la equidad, la participación comunitaria y el trabajo intersectorial. En este sentido, las acciones de promoción de la salud se constituyen en procesos políticos y sociales, que buscan incidir en las condiciones ambientales, sociales y económicas; por tal motivo pretende: promover la responsabilidad social de la salud, incrementar la inversión para el desarrollo de la salud, consolidar y expandir nuevas alianzas para la salud, aumentar la capacidad de la comunidad, crear poder de los individuos (empoderamiento social) y asegurar una infraestructura para la promoción de la salud; en consecuencia, la promoción de la salud (PS) es considerada como un conjunto de acciones, con el fin de que el bienestar se convierta en la tarea social más importante, no solo del individuo, sino de la sociedad (Carmona, Rozo y Mogollón, 2005, p. 72).

Por su parte, el investigador Juan Carlos Eslava (2006, p. 108) considera que la promoción de la salud ha sido caracterizada desde tres acepciones diferentes, pero complementarias:

1. Como una orientación de política sanitaria a nivel internacional, que busca intervenir sobre el entorno y modificar los estilos de vida, posibilitando con ello que las opciones más saludables sean las más fáciles de elegir.

2. Como un conjunto de acciones y procesos encaminados a que las comunidades y los individuos estén en condiciones de ejercer un mayor control sobre los determinantes de la salud, manteniendo o mejorando, de ese modo, su estado de salud.

3. Como una dimensión del trabajo, que toma como eje la salud en sentido positivo $-\mathrm{y}$ no la enfermedad- $\mathrm{y}$ tiene por objeto mantener las condiciones $\mathrm{y}$ garantizar condiciones de bienestar de los individuos y las poblaciones.

Con base en estas acepciones, Eslava (2006) considera que es entendible por qué la promoción de la salud (PS) se ha planteado, a veces indiscriminadamente y sin una coherencia interna, como: una dimensión, estrategia, proceso y conjunto de acciones (pp. 7-9). Por tal razón, este autor menciona que en la actualidad la visión que tiene más peso es aquella que entiende a la PS como un referente general para las políticas de salud y estrategia fundamental de la acción sanitaria; pero al mismo 
tiempo, como estrategia, dado que se dirige a proporcionar a los pueblos los medios necesarios y suficientes para mejorar su salud y ejercer un efectivo control sobre esta.

De acuerdo a los planteamientos de los autores citados, existe una coincidencia entre el enfoque de la capacidad de potencializar el desarrollo humano y el señalado por Juan Carlos Eslava, dado que ambos se orientan a realizar acciones para el empoderamiento de las personas y las comunidades, a fin de potencializar lo positivo y ejercer control sobre los determinantes de la salud.

\section{LA NUEVA LÓGICA DE PROMOCIÓN DE LA SALUD, DESDE EL ÁMBITO SOCIAL}

Según lo anotado anteriormente, existen diferentes enfoques de promoción de la salud; sin embargo, en cuanto a su conceptualización, prevalece el establecido en la Carta de Otawa, en 1986, citado por Picado y Quesada (2010), donde se define como "proporcionar a los pueblos los medios necesarios para mejorar su salud y ejercer un mayor control sobre la misma. Trasciende la idea de formas de vidas sanas para incluir las condiciones y requisitos para la salud que son: paz, vivienda, educación, alimentación, renta, ecosistema estable, recursos sostenibles, justicia social y equidad". Constituye un proceso político y social global que abarca no solamente las acciones orientadas a fortalecer las habilidades y capacidades de los individuos, sino también las dirigidas a fortalecer las condiciones sociales, ambientales y económicas requeridas para impactar positivamente en la salud colectiva e individual.

Busca generar procesos de educación y acción social que procuran estimular la conciencia pública sobre el valor de la salud. A su vez, promueve el desarrollo de condiciones y estilos de vida saludables y fomenta la participación organizada de la comunidad e instituciones públicas y privadas.

Para orientar y direccionar las actuaciones de las instituciones vinculadas a la promoción de la salud como parte de sus responsabilidades en la producción de la salud, se establecen las siguientes cinco líneas de acción (ver Tabla 1).

De igual forma, se definieron una serie de componentes y herramientas básicas de actuación. Entre las herramientas se tiene: educación para la salud (formal e informal), comunicación social, la intersectorialidad, las alianzas estratégicas, las redes de apoyo social, la abogacía de la salud y la investigación (Ministerio de Salud, 1999, p. 170).

Pese a que se ha reconocido la trascendencia del enfoque de promoción de la salud, han existido dificultades para hacerlo operativo, dado que este término se asocia con diversas formas de entenderlo, además del predominio del paradigma 


\section{Tabla 1}

LAS LÍNEAS DE ACCIÓN DEL ENFOQUE EN PROMOCIÓN DE LA SALUD

\section{LÍNEAS DE ACCIÓN}

\section{Elaboración de políticas públicas} saludables

Fortalecimiento de la participación social

\section{Fortalecimiento de habilidades y potencialidades individuales y colectivas}

Creación de ambientes saludables

Reorientación de los servicios de salud
Se refiere al conjunto de políticas públicas como las leyes, decretos, reglamentos o normas establecidas desde los sectores públicos con repercusiones relevantes a corto y largo plazo en el estado de salud de la población.

Se refiere al proceso de construcción y producción de la salud, en el cual intervienen tanto las instituciones y órganos del sector de la salud como los demás sectores relacionados con él, en los ámbitos público y privado. Permite a las personas, grupos organizados e instituciones tener los conocimientos, la capacidad, la información y el poder suficientes para garantizarle a la colectividad condiciones para una vida sana y el acceso equitativo a bienes y servicios.

Proceso que promueve la habilidad para tomar decisiones y ganar control sobre la vida personal, lo cual tiene que ver con la percepción de la competencia y eficacia. Para ello, se debe empoderar a las personas y a las colectividades para que tomen decisiones informadas acerca de su salud, asimismo requiere de la socialización del conocimiento técnico científico en salud.

Proceso que promueve las acciones que consideren todos aquellos aspectos identificables del entorno: físico, social, emocional, económico, ecológico y político saludable que influencian la salud.

La salud humana se construye según la forma en que tratamos a la naturaleza y a nuestro propio ambiente (educativo, laboral, familiar, socialnatural).

Priorizar la actuación de los servicios sobre los determinantes de la salud (conciencia y conducta, económico-ecológico, biológico), más que sobre los daños que estos ocasionan. Proceso de adaptación de sus estructuras y funciones a las nuevas demandas de salud. Implica alianzas con otros sectores para influenciar en la atención integral de factores que condicionan la salud. 
biologista-médico-asistencialista de las instituciones y organizaciones que producen servicios en salud; es decir, las acciones siguen orientándose a la prevención de la enfermedad. Para una mejor comprensión en relación con las diferencias entre ambos enfoques se presenta la Tabla 2, que permite distinguir las diferencias en ambos enfoques.

\section{Tabla 2}

PRINCIPALES DIFERENCIAS ENTRE PROMOCIÓN DE LA SALUD Y PREVENCIÓN DE LA ENFERMEDAD

\section{PROMOCIÓN DE LA SALUD}

Visión salutogénica

Modelo social

Actuación sobre determinantes y factores protectores de la salud

Aplicación de métodos, técnicas y estrategias para favorecer las condiciones salutogénicas

Se dirige a población en general como comunidades o grupos específicos (niñez, adolescentes, adultos, mujeres, entre otros)

Enfoque de derechos

Busca proteger y fortalecer la salud

Fortalecimiento de procesos de participación social intersectorial-política

Amplia cobertura y beneficia a mayor número de personas o población

Busca desarrollar potencia, potencialidades positivas y fortalezas individuales y colectivas (gruposcomunidad-nacional)

Acciones de educación e información en salud hacia el reforzamiento de comportamientos para el logro de condiciones y estilos de vida saludables

Busca mejorar las condiciones y calidad de vida de la población. Sus resultados son de largo plazo

\section{PREVENCIÓN DE LA ENFERMEDAD}

\author{
Visión patogénica
}

Modelo biomédico-enfoque asistencial

Actuación sobre factores de riesgo

Aplicación de técnicas para reducir los factores de riesgo y enfermedad o daño

Primaria: se dirige a personas y grupos de riesgo Secundaria: personas o grupos posiblemente enfermos (tamizajes)

Terciaria: enfermos en riesgo de complicaciones y muerte

Privilegia solo grupos de riesgo

Disminuir complicaciones de enfermedad

Débil participación en escenarios políticos e intersectoriales, centrada en comunidades de riesgo. Acciones puntuales

Cobertura limitada, se centra en personas afectadas. Es focalizada

Aplicación de técnicas para evitar la aparición o avance de una enfermedad o daño

Acciones de educación e información hacia la protección de los individuos de los factores de riesgo o problemas y sus posibles causas, busca modificación de conductas de riesgo

Busca disminuir riesgos por lo que su protección es específica. Resultados limitados a corto plazo

Fuente: Elaboración propia y ajustada al enfoque positivo, a partir de diversas fuentes documentales internacionales y nacionales consultadas, referentes al tema de promoción de la salud (Picado y Quesada, 2010). 
De acuerdo a la Tabla 2, se puede observar que la Promoción de la Salud, representa un modelo social que, mediante sus métodos e instrumentos, busca innovar la forma de trabajar de sectores y actores sociales, a fin de abordar los determinantes generadores de salud y fomentar el cambio hacia una verdadera cultura de salud. De igual forma, propicia el diseño de estrategias dirigidas a establecer criterios de funcionabilidad en la evaluación del impacto de las acciones. Por el contrario, la Prevención de la Enfermedad limita su actuación sobre los factores y grupos de riesgo, por lo que su acción es focalizada y excluyente con escasos productos y resultados. El primero integra los derechos en general, mientras que el segundo solo para algunos grupos específicos.

En correspondencia con lo mencionado, el investigador Juan Carlos Eslava propone una nueva e innovadora lógica para abordaje del enfoque en promoción de la salud. Su propuesta se dirige a hacer un desplazamiento de la manera como se identifica en ¿Qué hacer?, en un Programa, Proyecto, Servicio o Actividad; es decir, en lugar de plantearse un problema en salud, definido como enfermedad, el discurso de la PS procura formular objetivos de satisfacción (o satisfactores) a alcanzar y no se dirige a identificar factores de riesgo, sino busca identificar las condiciones necesarias para alcanzar estos objetivos (o satisfactores) (Eslava, 2002).

Como se visualiza en la Figura 1, se establecen una serie de preguntas generadoras que permiten guiar y organizar las acciones en promoción de la salud. Se parte de la interrogante ¿Qué se desea hacer?, esto para tener mayor claridad

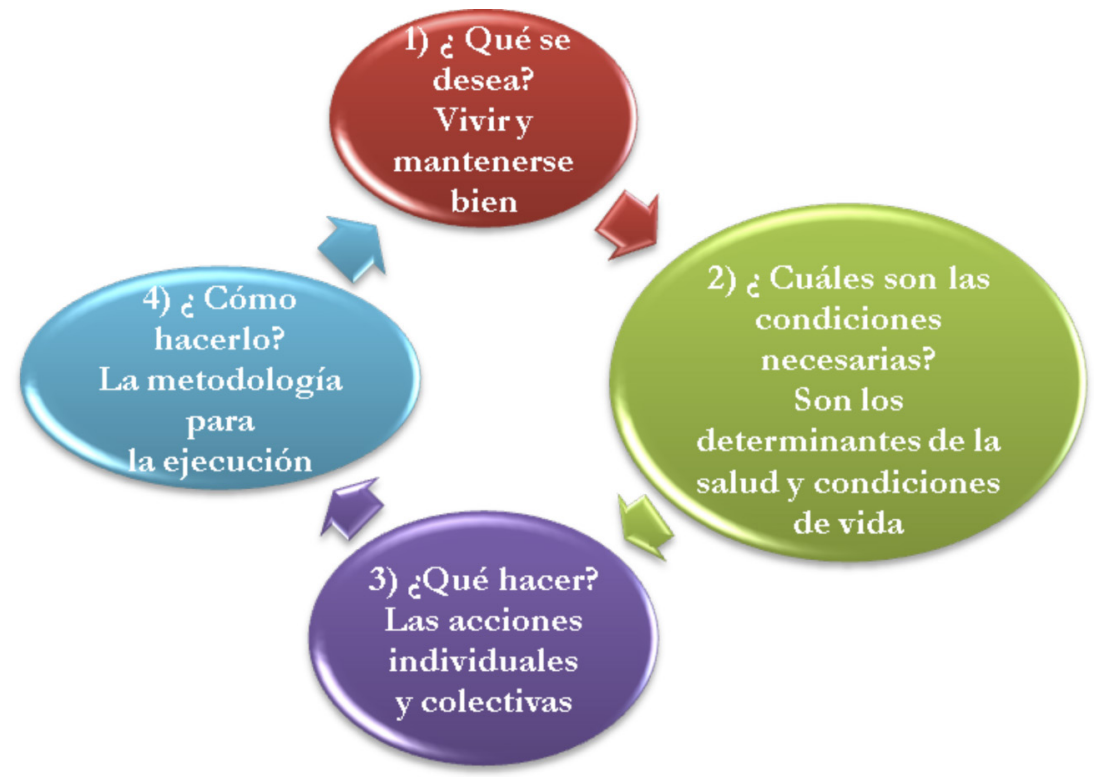

Figura 1. Lógica de abordaje desde el enfoque en promoción de la salud. Fuente: (Eslava, 2002). 
sobre lo que se quiere alcanzar; es decir, permite definir los objetivos óptimos de la salud, que buscan identificar, desarrollar y fortalecer los factores o aspectos positivos existentes, para posteriormente definir las condiciones necesarias para realizar acciones que permitan su alcance. Con la tercera pregunta, se concretan las actividades necesarias a realizar, tanto a nivel individual como colectivo. El cuarto momento corresponde a la metodología a utilizar.

En congruencia con estos planteamientos, las autoras del presente artículo han considerado la importancia de proponer la integración de los enfoques citados para darle la integralidad y efectividad a las actuaciones desde la perspectiva de promoción de la salud; es decir, los de políticas públicas y desarrollo humano, a partir de la nueva lógica de trabajo más positiva, proactiva, propositiva e innovadora para impactar favorablemente en las condiciones, calidad y estilos de vida y sus diversos determinantes desde el escenario de salud.

\section{¿Por qué promoción de la Salud con enfoque positivo, proactivo, propositivo e inno- vador?}

Para dar respuesta a esta pregunta, se dará una justificación a cada uno de los aspectos integrados en ella.

Positivo: parte de la identificación, priorización de factores protectores de la salud, según determinantes sociales, económicos, ambientales, culturales, políticos, entre otros. Es mirar, planificar y actuar a partir de lo que está bien, trabajar potenciando y desarrollando procesos vistos desde las posibilidades y no carencias, desde las fortalezas y oportunidades en los ámbitos personales, grupales y de sociedad civil en general.

Propositivo: revisión y diseño de propuestas, alternativas o iniciativas que contribuyan a la toma de decisiones en cuanto al posicionamiento del nuevo paradigma de salud, del enfoque de interés de este artículo y generación de la cultura de salud desde la lógica positiva. Constituye procesos que deben estar acordes a los constantes cambios.

Proactivo: al actuar sobre lo positivo, sobre lo que está bien, implica la capacidad de anticiparse y desarrollar procesos necesarios para contribuir a que dichas condiciones positivas se mantengan y fortalezcan. Incluye iniciativa, emprendedurismo y perseverancia, lectura constante del entorno, hacer proyecciones y tomar decisiones previas, es decir: antes de.

Innovador: las innovaciones sociales deben ser sociales, tanto en sus medios como en sus fines y se definen como nuevas ideas (productos, servicios y modelos) que simultáneamente dan respuesta a las necesidades sociales y crean nuevas relaciones sociales o colaboraciones generando bienestar en la sociedad y al mismo 
tiempo produciendo mejora en su capacidad para actuar (Agnès Hubert, citado en Edwards, 2012).

Con la fundamentación teórica expuesta anteriormente, se construyeron y se ejecutan dos proyectos de extensión docente que fomentan un proceso de educación continua, con diversos actores sociales de diversos sectores e instituciones (públicas y privadas) de la Región de Occidente de Costa Rica. El proceso de estos dos proyectos se presenta a continuación.

\section{EL PROCESO DE EDUCACIÓN CONTINUA EJECUTADO PARA LA EFECTIVA APLICACIÓN DEL ENFOQUE EN PROMOCIÓN DE LA SALUD}

\section{¿Cuál fue la realidad regional que motivó generar el proceso de educación continua?}

Como parte de un proceso internacional, nacional y luego regional, en el año 2004 se realizó, en la Región de Occidente de Costa Rica, la medición de las funciones esenciales de salud pública, cuya conducción estuvo a cargo del Ministerio de Salud con el apoyo de la Organización Panamericana de Salud. Los resultados obtenidos en esta primera medición representaron un insumo básico para determinar el proceso de planificación y acciones tendientes a mantener las funciones en salud.

La medición abarcó 11 funciones esenciales, pero dos de ellas fueron de vital interés para las autoras de este artículo, dado que hacían referencia a la manera como se estaba integrando el enfoque de promoción de la salud en el contexto regional y local. Estas funciones fueron: promoción de la salud y desarrollo de recursos humanos y capacitación en salud pública.

Con respecto a la función en promoción de la salud, los resultados demostraron que son evidentes los esfuerzos desarrollados por la autoridad sanitaria regional de Occidente, para avanzar en los procesos con este enfoque, sobre todo en lo correspondiente a reorientar los servicios hacia la adopción de una nueva cultura de salud, donde se potencializan y maximizan los factores protectores de la salud, que procura fomentar conductas y ambientes saludables; sin embargo, también se reconoció que algunas instituciones, si bien indicaban impulsar acciones de promoción de la salud, al ejemplarlas, se determinaba confusión pues realmente eran de prevención de la enfermedad. Por ejemplo: desarrollar actividades relacionadas con estilos de vida saludable dirigidas a grupos de crónicos; es decir, grupos de riesgo para prevenir enfermedades crónicas no trasmisibles, hallazgo que lógicamente ameritaba clarificación conceptual y de enfoque. En este contexto se han facilitado: 
capacitaciones, asesorías, normas, intervenciones, así como el establecimiento de alianzas estratégicas y planificación intersectorial hacia la unificación de criterios y consecución de metas en pro de la salud pública. La función del desarrollo de recursos humanos y capacitación en salud pública fue el de menor desarrollo, específicamente con el indicador que midió el perfeccionamiento de los recursos humanos.

A raíz de los resultados emitidos en esta medición, se conformó la Comisión Interinstitucional de Promoción de la Salud de la Región (CRIPS), la cual elaboró un Primer Plan Estratégico de Promoción de la Salud, 2005-2007 y luego otro plan para el periodo 2008-2011. En el marco de estos planes se realizaron diversas actividades, tales como: a) El 23 de noviembre de 2004 se realizó un Taller Inter-organizacional; b) Para el año 2007, la Comisión programó un taller sobre "Las políticas públicas locales en salud con enfoque de género y promoción social”, coordinado por el Ministerio de Salud, el Instituto Nacional de la Mujer, la Sede Regional de Occidente de la Universidad de Costa Rica y Federación (FEDOMA); c) El 4 de noviembre de 2008 se ejecutó un Foro Regional titulado "Promoción de la Salud y Prevención de Enfermedad: dos enfoques distintos pero complementarios".

Producto de dichas actividades, los participantes expusieron como recomendaciones para la Comisión Intersectorial: 1) Redireccionar el enfoque de prevención vs. promoción, de los profesionales de la salud; 2) Dotar de talento humano necesario a las Áreas de Salud de la CCSS, para implementar la promoción de salud; 3) Continuar realizando este tipo de actividades, para que exista más conocimiento alrededor del tema, y 4) Sensibilizar a funcionarios, estudiantes y personas de la comunidad, acerca de la especificidad de la promoción de la salud.

Al tomar en consideración estas recomendaciones, la Región Rectora de Salud y la carrera de Trabajo Social de la Sede de Occidente, Universidad de Costa Rica, realizaron dos actividades sustantivas: un proyecto de investigación titulado "La Promoción de la Salud en el contexto de las instituciones públicas de la Región Central Occidental de Costa Rica" ( $\left.N^{\circ} 540-A 9-018\right)$ y un proceso de educación continua que se manifestó en dos proyectos de extensión docente. Seguidamente, se expone las características principales de los proyectos de extensión docente.

\section{Primer proyecto: "Fortaleciendo las capacidades humanas y técnicas de los actores socia- les de la Región Central Occidente, para la efectiva aplicación del Enfoque en Promoción de la Salud".}

El primer proyecto se planteó como meta para el año 2009-2010 ejecutar un proceso de educación continua dirigido al personal de las Áreas Rectoras de Salud 
del Ministerio de Salud, de las Áreas de Salud de la CCSS, docentes y estudiantes universitarios de la Sede de Occidente, Universidad de Costa Rica (recintos de San Ramón y Tacares), funcionarios de instituciones de otros sectores y actores comunales de la región.

Este proceso de educación continua tenía como objetivo general: "Propiciar las condiciones necesarias para el fortalecimiento de las capacidades humanas y técnicas de los actores sociales de la Región de Occidente, con el propósito de avanzar hacia la efectiva aplicación del Enfoque de Promoción de la Salud"; el cual se ejecutó de setiembre de 2009 a junio de 2010, se contó con la participación de los siguientes actores sociales: Ministerio de Salud: Áreas Rectoras de Salud, Sarchí, Zarcero, Naranjo, San Ramón y Atenas; Ministerio de Educación: Región Educativa Occidente; Caja Costarricense de Seguro Social: Áreas de Salud San Ramón, Naranjo, Clínica Clorito Picado; Universidad de Costa Rica, Sede Occidente: carrera de Trabajo Social y Consultorio Social; Municipalidades: Naranjo y Atenas; ONG: Mujeres Unidas en Salud y Desarrollo (MUSADE) y Comité Cantonal de Deportes San Ramón.

El proceso de capacitación se ejecutó con una metodología constructivista, pues los actores trabajaron aspectos teóricos y metodológicos del enfoque en promoción de la salud, a la luz de las experiencias e iniciativas de los escenarios que representaba cada actor participante. Cabe destacar que se ejecutaron actividades educativas de forma sincrónica (presenciales) y asincrónicas (a distancia).

Las actividades de capacitación se realizaron en cinco momentos, complementarios entre sí:

- Primer momento: "Curso-Taller sobre fundamentos teóricos-metodológicos para formular proyectos con enfoque de promoción de la salud"

Este curso inició en la Sede de Occidente, Universidad de Costa Rica, el 3 y 4 de setiembre de 2009, el cual fue dirigido a un grupo conformado por 30 personas representantes de diferentes instituciones. Durante los dos días se efectuaron actividades de trabajo grupal, que permitieron el intercambio de conocimientos y experiencias sobre el tema, entre las personas participantes, a fin de que lograran identificar las prioridades de promoción de la salud en sus escenarios de actuación y con base en esto formularan una idea de proyecto con enfoque.

Para efectos del curso, las facilitadoras ajustaron una metodología de identificación y formulación de proyectos, acorde a la nueva lógica de trabajo del enfoque en cuestión.

En este momento, se identificaron 12 ideas de proyectos, propuestos por equipos de trabajo inter-institucionales. 
- Segundo momento: "Intercambio a distancia de los avances y retroalimentación de los proyectos formulados"

Este momento consistió en habilitar espacios de intercambio presenciales y a distancia, con el objetivo de que las personas participantes compartieran el proceso de formulación de sus proyectos con enfoque en promoción de la salud. Se contó con la asistencia e involucramiento continuo y sistemático de 27 personas. Entre los logros, destacan el análisis del marco legal internacional, nacional e institucional que da sustento a las acciones de promoción de la salud, de igual manera, la formulación de 10 perfiles de proyectos de promoción de la salud de índole intersectorial.

- Tercer momento: "Curso-Taller sobre los fundamentos teóricos-metodológicos para la evaluación de los proyectos con enfoque de promoción de la salud"

Este momento consistió en el desarrollo de un taller de tres días, que tuvo como objetivo "Facilitar fundamentos teóricos-metodológicos, para la evaluación de los proyectos con enfoque de promoción de la salud, implementados por los actores sociales participantes, en la Región de Occidente".

Con dichas actividades educativas, se logró diseñar matrices para la evaluación de los respectivos proyectos en promoción de la salud.

- Cuarto momento: "Jornada de presentación de resultados de proyectos implementados por los actores sociales"

Como última actividad educativa, se ejecutó una jornada de presentación de resultados de proyectos, a fin de proyectar y validar los logros y alcances del proceso de capacitación, así como retroalimentar la experiencia a otros actores sociales, no solo de la Región de Occidente, sino también a otras regiones del país.

Al final del proceso se pudieron contabilizar los siguientes productos generales: 27 personas capacitadas, 8 instituciones participantes, 10 proyectos de promoción de la salud.

- Quinto momento: "Sistematización del proceso de educación continua"

Este momento consiste en la elaboración de un informe de sistematización de la experiencia vivida, a fin de realimentar futuros proyectos de esta naturaleza. 
Segundo proyecto: "Fomentando la cultura de Salud desde el enfoque de Promoción de la Salud: Una acción hacia la integración de actores y redes sociales"

Este segundo proyecto inició el 3 de junio y finalizó la primera semana de diciembre de 2011. El objetivo general: "Fomentar procesos de asesoría técnica, capacitación y comunicación social sobre el Enfoque en Promoción de la Salud para la creación de una red de actores sociales de la Región Central Occidental, así como en otras regiones de Costa Rica, a fin de posicionar la cultura de la salud desde el enfoque de promoción de la salud". Dos de los objetivos específicos fueron: "Establecer espacios de capacitación para actores sociales intersectoriales, que permitan la adquisición y actualización de conocimientos relativos al enfoque de promoción de la salud desde la perspectiva proactiva, positiva e innovadora, para el desarrollo de sus habilidades individuales y grupales que les faciliten la aplicación del enfoque en cuestión" y "Facilitar la integración de redes sociales en la Región Central Occidental, para la consolidación y continuidad de los procesos de promoción de la salud desarrollados tanto a nivel institucional como intersectorialmente en la Región".

Originalmente, este proyecto se delimitó geográficamente en la provincia de Alajuela; sin embargo, se extendió a otras provincias (San José, Heredia y Puntarenas), por demanda de actores sociales de instituciones de dichos lugares.

Las instituciones participantes en este segundo proceso fueron:

- $\quad$ Ministerio de Salud: Encargadas de Mercadoctenia de la Salud/RCO

- Universidad de Costa Rica, representante de la carrera de Trabajo Social

- $\quad$ Municipalidades: Zarcero y Atenas de la Región Central de Occidente

- Caja Costarricense de Seguro Social

- Instituto Nacional de Aprendizaje

Se contó con la participación de 32 actores.

Este proyecto se ejecutó, también, con una metodología constructivista, donde los actores sociales involucrados se constituyeron en protagonistas del desarrollo de las diferentes etapas del proyecto.

- Primer momento: Promoción y divulgación del proyecto con actores sociales a involucrar

Para iniciar el proyecto se realizaron actividades de divulgación e información con actores sociales egresados del I Proyecto de Extensión Docente, así como con otros actores que mostraron interés en integrarse. 
- Segundo momento: Capacitación de los actores sociales sobre el Enfoque en Promoción de la Salud

Después de haber realizado un proceso de inscripción, se empezó a impartir un curso de capacitación a distancia, por medio de la plataforma de Mediación Virtual de la Universidad de Costa Rica; inició con una sesión presencial que permitió exponer las bases conceptuales del curso y brindar las indicaciones para el uso de esta herramienta informática.

\section{- Tercer momento: Asesoría a actores sociales sobre el Enfoque en Promoción de la Salud}

Como parte del proceso de capacitación se brinda asesoría individual y grupal, ante inquietudes y demandas específicas de las personas participantes.

\section{- Cuarto momento: Consolidación de una red de actores sociales}

Para este momento, se motivó e impulsó la integración de actores sociales mediante una red, que defina un plan de trabajo en conjunto, que facilite la continuidad de planes, programas, proyectos y actividades regionales con enfoque en promoción de la salud.

\section{CONCLUSIONES}

El siguiente artículo nos permite concluir lo siguiente:

Considerando el desarrollo teórico del concepto de salud-enfermedad, es imprescindible fomentar espacios de análisis colectivo sobre el significado de salud que emerge en los servicios de salud, a fin de generar las herramientas necesarias a los actores sociales, para que logren trascender el paradigma biologista, pues contradictoriamente abordan la salud desde la enfermedad, dejando de lado las condiciones salutogénicas. De igual forma, se visibiliza el enfoque de promoción de la salud, a partir de una visión positiva, proactiva e innovadora, como uno de los ejes centrales en la construcción social de la salud.

Para que haya un efectivo posicionamiento de la promoción de la salud desde la visión positiva, proactiva e innovadora, se requiere no solamente identificar sus diferencias con respecto a la prevención de la enfermedad, sino también mantener claridad teórico-metodológica y operativa respecto a este enfoque. Se necesita fomentar un proceso permanente de lectura y análisis del entorno local, regional, 
nacional e internacional, identificando con ello los determinantes y factores protectores de la salud, para definir las estrategias, los objetivos de satisfacción y planificar acciones colectivas que actúen sobre aquellos y así impactar favorablemente como ya se ha señalado, sobre las condiciones y calidad de vida en la población, integrando las propuestas del enfoque, específicamente las políticas públicas, el desarrollo humano y la nueva lógica de trabajo de promoción de la salud expuestas en este artículo.

Las experiencias desarrolladas en la Región Central Occidental de Costa Rica, demuestran la factibilidad de articular esfuerzos, recursos y talentos entre Universidades e Instituciones Públicas, para generar procesos de educación continua, que contribuyan a llenar los vacíos, fortalecer debilidades y atender los intereses que presentan los actores sociales. Por otra parte, las experiencias son una contribución de cómo hacer efectivas las intensiones técnico-políticas en el caso particular de la promoción de la salud, aprovechando el predominio de múltiples factores protectores, la respuesta positiva y los procesos de participación social existentes en la región que posibilitan su implementación. Todo lo cual ha permitido dar continuidad a los procesos de posicionamiento del enfoque señalado, sobre todo con gobiernos locales, Ministerio de Educación y Caja Costarricense de Seguro Social, especialmente en la Región Central Norte, los cuales se orientan a la construcción de una verdadera cultura de salud.

Como principales desafíos de trabajo en el campo de la promoción de la salud, se pueden considerar los siguientes:

- Trascender los paradigmas prevalentes sobre salud-enfermedad en autoridades políticas y técnicas, así como en los servicios sociales y la sociedad en general, quienes privilegian con sus políticas y planificación las actuaciones institucionales y comunidades que fomentan acciones focalizadas en factores de riesgos y problemas; al tiempo que excluyen las poblaciones y condiciones positivas y salutogénicas existentes, que también deben ser visibilizadas en los procesos de planificación estratégica en salud, en todas sus etapas metodológicas, para mantener y fortalecer dicha condición. Esto posibilita que se invierta en salud y no solo se gaste en la atención de la enfermedad, que se trabaje realmente con enfoque de derechos, de género e inclusión. En este caso, es fundamental que a nivel país se impulse con claridad el abordaje del proceso salud-enfermedad desde la visión social, donde se dé prioridad a la implementación del enfoque de promoción de la salud ya planteado.

- Definir mecanismos y estrategias que permitan mantener el proceso continuo y sostenido de la promoción de la salud, para formar adecuadamente a los y las profesionales en este ámbito. 
- Definir un perfil para los profesionales que desempeñan funciones y desarrollen las correspondientes acciones.

- Además, evaluar las intervenciones, acciones y componentes que realizan las instituciones con respecto al enfoque, a fin de que los resultados permitan un fortalecimiento de su aplicación.

Se visualiza que en futuros proyectos de educación continua se generen talleres de estrategias de comunicación social para el desarrollo en promoción de la salud, ya que busca darle mayor contenido a la comunicación ubicándola en los procesos culturales, sociales y políticos, recuperando su sentido educativo-participativo, apostando por una comunicación ciudadana vinculada a gestar procesos de desarrollo que se articulen a la vida cotidiana de la gente, y que orienten la acción ciudadana hacia el cambio social.

Asimismo, facilitar tecnologías para el intercambio de información, capaces de generar realimentación y el fortalecimiento de actitudes, prácticas y hábitos orientados hacia una verdadera cultura en salud.

\section{REFERENCIAS}

Breilh, J. (1994). Nuevos conceptos y técnicas de investigación guía pedagógica para un taller de metodología. Serie Epidemiología Crítica Número 3. (1ed.). Ecuador: Ediciones CEAS.

Carmona, L., Rozo, C. y Mogollón, A. (2005). La Salud y la Promoción de la Salud: una aproximación a su desarrollo histórico y social. Revista Ciencias de la Salud,3(1), 62-77. Recuperado de http://www.redalyc.org/articulo.oa?id=56230108/

Eslava, J.C. (2002). Promoción y Prevención en el Sistema de Salud en Colombia. Revista de Salud Pública, 4(1), 1-12. Recuperado de http://www.scielo.org.co/scielo.php?script=sci_ arttext\&pid=S0124-00642002000100001\&lng=en\&tlng=es/

Eslava, J.C. (2006). Repensando la promoción de la salud. Revista de Salud Pública, 8(Su2), 106-115. Recuperado de http://www.redalyc.org/articulo.oa?id=4220990/

Ministerio de Salud. (1999). Plan Nacional de Promoción de la Salud 1999-2004. Costa Rica: Ministerio de Salud.

Ministerio de Salud. (2004). Medición Regional de las Funciones Esenciales de Salud Pública en la Región Central Occidente. Costa Rica: Unidad Regional de Desarrollo de la Salud, Ministerio de Salud. 
Edwards, M. (2012, setiembre 7). ¿Qué es innovación social?. [Registro web]. Recuperado de http:// www.tendencias21.net/innovacion/Que-es-la-innovacion-social_a45.html/

Picado, L. y Quesada, A. (2009). Propuesta de capacitación continua: “Fortaleciendo las capacidades humanas y técnicas de los actores sociales de la Región de Occidente hacia la efectiva aplicación del Enfoque de Promoción de la Salud”. [Proyecto de Extensión Docente]. Región Rectora de la Salud Occidente y Universidad de Costa Rica, Sede de Occidente.

Picado, L. y Quesada, A. (2010). “Aclarando las posiciones teóricas sobre el Enfoque en Promoción de la Salud: en busca de su efectiva implementación en el contexto actual". Revista Trabajo Social, (12), 73-90.

\section{SOBRE LAS AUTORAS}

Ana Cristina Quesada Monge: Trabajadora Social, docente universitaria. Máster en Trabajo Social, énfasis en Gerencia Social. Carrera de Trabajo Social, Sede de Occidente, Universidad de Costa Rica. Correo electrónico: cristina.quesada@ucr. ac.cr

Lidia Isabel Picado Herrera: Trabajadora Social. Máster en Salud Pública, énfasis en Políticas de Salud. Promoción de la Salud, Unidad de Rectoría de la Salud, Región Rectora de la Salud de Occidente, Ministerio de Salud. Correo electrónico: lpicadoherrera@gmail.com / lidiai5@yahoo.es 
